\title{
DINÂMICAS ORÇAMENTÁRIAS DOS GOVERNOS LOCAIS PARANAENSES: SEGUINDO O INCREMENTALISMO OU EQUILÍBRIO PONTUADO?
}

\author{
Budgetary dynamics of local governments in Paraná: Following \\ incrementalism or punctuated balance?
}

Cristiane Aparecida da Silva

E-mail: cristianedasilva@ufgd.edu.br

Mestra e Doutoranda em Contabilidade pela Universidade Federal de Santa Catarina; Contadora na Universidade Federal da Grande Dourados. Endereço para contato: Centro Socioeconômico, Campus Universitário, Trindade, 88040-970,

Florianópolis, Santa Catarina, Brasil. https://orcid.org/0000-0003-4052-4231

Ricardo Suave

E-mail: ricardosuave@outlook.com

Doutor em Controladoria e Contabilidade pela Faculdade de Economia, Administração e Contabilidade da Universidade de São Paulo, com período sanduíche na University of Illinois at Urbana-Champaign nos Estados Unidos; Mestre em Contabilidade pelo Programa de Pósgraduação em Contabilidade da Universidade Federal de Santa Catarina; Professor no Curso de Ciências Contábeis da Universidade Estadual do Centro-Oeste de Guarapuava. https://orcid.org/0000-0002-9149-2658

Fabiano Aparecido da Silva E-mail: fabianocontabilista@bol.com.br Especialista em Contabilidade Pública Pela Uninter; Graduado em Ciências Contábeis pela Fatecie; Contador na Câmara Municipal de Miraselva. https://orcid.org/0000-0001-6248-5144

Fabricia Silva da Rosa

E-mail: fabriciasrosa@hotmail.com

Pós-doutorada em Contabilidade pela Universidade Federal de Santa Catarina; Doutora em Contabilitad com período sanduíche na Universitat de València; Doutora e Mestra em Engenharia de Produção pela Universidade Federal de Santa Catarina; Professora adjunta na Universidade Federal de Santa Catarina e no Programa de Pós-graduação em Contabilidade da Universidade

Federal de Santa Catarina. https://orcid.org/0000-0003-4212-1065

Artigo recebido em 9 de fevereiro de 2020. Aceito em 15 de julho de 2020. 


\section{Resumo}

Este estudo objetivou analisar se o processo orçamentário dos governos locais paranaenses segue os padrões de mudança incremental nas dotações orçamentárias ou ocorrência de pontuações orçamentárias. No que se refere aos objetivos, este estudo se caracteriza como descritivo. Quanto à abordagem, a pesquisa se caracteriza como quantitativa. A amostra corresponde a 39 governos locais paranaenses, cujo levantamento de dados diz respeito ao eixo temporal 2014 a 2017. Os dados foram coletados no site do Sistema de Informações Contábeis e Fiscais do Setor Público Brasileiro (Siconfi) em outubro de 2018. Foram estabelecidas quatro variáveis de análise: educação, saúde, segurança pública e urbanismo. Levando-se em consideração os aspectos analisados, conclui-se que a variação percentual nos dados de apropriação do orçamento é distribuída de forma não linear, conforme ilustrado pelas apresentações gráficas e estatísticas descritivas, sugerindo a ocorrência de um padrão de equilíbrio pontuado e também a ocorrência de pontuações simultâneas nas alocações orçamentárias entre os setores das políticas públicas. As limitações da pesquisa são concernentes à seleção da amostra e variáveis analisadas. Como apontamentos de pesquisas futuras, sugere-se utilizar um recorte longitudinal maior, bem como a aplicação de modelos com testes estatísticos.

Palavras-chave: Dinâmica orçamentária. Incrementalismo. Equilíbrio pontuado. Execução orçamentária.

\section{Abstract}

This study aimed to analyze whether the budget process of Local Governments in Paraná follows the patterns of incremental change in budget allocations or occurrence of budget scores. Regarding the objectives, this study is characterized as descriptive. As for the approach, the research is characterized as quantitative. The sample corresponds to 39 local governments in Paraná, whose data collection concerns the time axis 2014 to 2017. The data were collected on the Brazilian Public Sector Accounting and Tax Information System (Siconfi) website in October 2018. Four were established analysis variables: education, health, public security and urbanism. Taking into account the analyzed aspects, it is concluded that the percentage variation in the budget appropriation data is distributed in a non-linear way, as illustrated by the graphic presentations and descriptive statistics, suggesting the occurrence of a punctuated equilibrium pattern and also the simultaneous scores in budget allocations between public policy sectors. The research limitations are related to the sample selection and analyzed variables. As notes for future research, it is suggested to use a larger longitudinal cut, as well as the application of models with statistical tests.

Keywords: Budgetary dynamics. Incrementalism. Punctuated balance. Budget execution.

\section{INTRODUÇÃO}

O processo contínuo de negociação social e política converge para a definição da forma como os recursos públicos serão aplicados na implementação de políticas públicas. Dessa forma, o orçamento público representa um movimento mais complexo do que a simples previsão de receitas e definição de despesas (Carvalho, 2018).

O governo local prepara o orçamento anual, alocando recursos disponíveis para diferentes setores, visando promover o bem-estar dos cidadãos. Os montantes reais alocados 
a diferentes áreas são determinados pela intenção e atenção do governo com relação a cada uma delas, conforme refletido nas respectivas políticas (Guragain \& Lim, 2018).

Tais alocações orçamentárias não são constantes ao longo do tempo; portanto, as mudanças anuais nas alocações são de grande interesse em estudos empíricos. As teorias do incrementalismo (Wildavsky, 1964) e do equilíbrio pontuado (Baumgartner \& Jones, 1993, 2010) oferecem explicações plausíveis para alterações nas dotações e políticas orçamentais nas respectivas funções orçamentais.

De acordo com a teoria do incrementalismo, em razão de restrições durante o processo de tomada de decisão, as mudanças nas políticas são graduais, com pequenas mudanças nas políticas existentes (Guragain \& Lim, 2018). Parte-se do pressuposto que cada orçamento público é elaborado com base no anterior, com pequenas variações, que são apenas incrementais. Portanto, no incrementalismo, a base do orçamento é tida como quase totalmente dada, sendo a sua principal preocupação compreender os fatores que explicam como ocorrem as mudanças marginais dessa base (Alves, 2016).

Baumgartner e Jones (2010) explicam que uma quebra no monopólio da política, desatenção em longo prazo e reação exagerada a alguma questão levam a mudanças políticas em larga escala em momentos específicos. Os autores definiram essas mudanças "episódicas" como "a teoria do equilíbrio pontuado", caracterizando, assim, a estabilidade em longo prazo e a mudança rápida em momentos específicos.

Com base nessas teorias, destacam-se alguns estudos empíricos que foram realizados para investigar a dinâmica do processo orçamentário em diferentes períodos, como os estudos de Carvalho (2018), Guragain e Lim (2018) e Silvestre e Araújo (2015). A partir dessa contextualização, apresenta-se como questão de pesquisa: as dinâmicas orçamentárias dos governos locais paranaenses seguem o incrementalismo ou equilíbrio pontuado? Para tanto, o objetivo do estudo é analisar se o processo orçamentário dos governos locais paranaenses segue os padrões de mudança incremental nas dotações orçamentárias ou a ocorrência de pontuações orçamentárias.

Este estudo se justifica ao estender a literatura acerca da análise empírica concernente ao orçamento público, sobretudo com a utilização da lente teórica do incrementalismo e do equilíbrio pontuado para avaliar o comportamento das variáveis orçamentárias como proxy de execução de políticas públicas. A partir disso, utilizam-se as variáveis de execução orçamentária (funções) como instrumento de mensuração da execução de políticas públicas, além de realizar uma análise teórica acerca dessas variáveis no contexto subnacional brasileiro.

Grande parte dos estudos orçamentários brasileiros é eminentemente técnico, não se utilizando de modelos teóricos para examinar e compreender o comportamento das decisões no processo orçamentário (Ollaik \& Medeiros, 2011). Conhecer as variedades de modelos teóricos existentes e compreender como se comporta a alocação dos recursos públicos são 
fatores que podem também contribuir para o aprimoramento da administração pública brasileira. Além da presente introdução, a estrutura do trabalho inclui fundamentação teórica, metodologia, resultados e discussão, e considerações finais.

\section{FUNDAMENTAÇÃO TEÓRICA}

\subsection{EQUILÍBRIO PONTUADO NO PROCESSO ORÇAMENTAL}

A Teoria do Equilíbrio Pontuado (TEP) foi desenvolvida por Frank R. Baumgartner e Bryan D. Jones em seu livro seminal Agendas e Instabilidade na Política Americana, baseado em estudos empíricos do processo político americano no final dos anos 1980 e início dos anos 1990 (Jones \& Baumgartner, 2012, p. 3).

Enquanto os arcabouços teóricos do processo de política costumavam enfocar a estabilidade, Baumgartner e Jones descobriram que a estabilidade não era permanente, sob a alegação de que as políticas tendem a ser estáveis por muito tempo e que essa estabilidade pode, às vezes, ser interrompida por mudanças políticas rápidas e substanciais (Kuhlmann \& Van Der Heijden, 2018).

A TEP analisa esses movimentos no processo das políticas públicas a partir de uma base teórica dupla, que contempla as instituições políticas e o processo decisório a partir da racionalidade limitada (Carvalho, 2018). A principal explicação para essa característica do processo de políticas públicas advém da interação de instituições políticas de diversos níveis e do processo decisório (Jones, True, \& Baumgartner, 2006).

Essa teoria enfatiza dois elementos relacionados ao processo de políticas, que são a definição do issue e o estabelecimento da agenda. Um issue ou questão de política é definido pelo discurso público e, na medida em que entra ou sai da agenda, definições de políticas públicas podem ser reforçadas ou questionadas (Jones et al., 2007).

De acordo com Givel (2010), a TEP aplicada na política pública é originária da teoria do equilíbrio pontuado biológico. $O$ autor afirma que a TEP no campo da biologia evolucionária ganhou força em 1954, quando Ernst Mayr sugeriu que evoluções não ocorrem gradualmente, mas com rápidas evoluções por meio de mutação e recombinação, por exemplo.

No âmbito do orçamento público, em contraste a teorias que buscam explicar ou a estabilidade ou a mudança, a TEP busca explicar ambas (Carvalho, 2018). Nesse sentido, segundo Carvalho (2018), a TEP permite analisar mudanças de larga escala que ocorrem em um processo estável da execução orçamentária, identificando, assim, períodos de equilíbrio e interrupção desse equilíbrio, bem como o melhor entendimento dessas mudanças bruscas. 


\subsection{INCREMENTALISMO NO PROCESSO ORÇAMENTAL}

A disputa pelas prevalências de demandas, quando da formulação do orçamento, conduz a um ponto de equilíbrio entre as reivindicações manifestas ao se reproduzir padrões decisórios anteriores. Essa lógica orçamentária é modificada apenas na ocorrência de eventos aleatórios de forte impacto para a (re)distribuição de recursos entre os grupos de interesses, quando o incrementalismo que a caracteriza é abandonado até que uma nova lógica incremental seja estabelecida em um novo patamar de alocação de recursos. $O$ processo incremental de alocação de recursos se inicia outra vez, até que um novo acontecimento de grande monta acirre o conflito distributivo (Fittipaldi, Costa, \& Araújo, 2017).

A definição operacional de "grande mudança" ou "pontuações" varia entre os estudiosos da literatura sobre o equilíbrio pontuado, ao passo que o grau de incrementalidade é explicado em diferentes faixas. Por exemplo, Wildavsky (1964) define a mudança incremental como uma mudança média anual de $10 \%$ na alocação do orçamento em comparação com o ano anterior. Bailey e O'Connor (1975) e Danziger (1976) definiram incrementalidade como apenas uma mudança positiva, enquanto outros autores incluíram mudanças negativas na sua definição.

Baumgartner e Jones (2010) utilizaram uma variação mais ampla, sendo esta de menos de $25 \%$ ou mais de $25 \%$ em relação ao ano anterior para explicar a pontuação. $O$ presente estudo utiliza esse intervalo por ser considerado o método convencional de análise de pontuação. A Tabela 1 resume o grau de incrementalidade explicado por diferentes estudiosos:

Tabela 1

Grau de incrementalidade

\begin{tabular}{lcc}
\multicolumn{1}{c}{ Fonte teórica } & Grau de incrementalidade & Versus \\
\hline Wildavsky (1964) & -10 a $10 \%$ & Ano anterior \\
Baumgartner e Jones $(1993,2010)$ & Mais de 25\%, menos de 25\% & Ano anterior \\
Bailey e O'Connor (1975) & Mais de 0\%, menos de 10\% & Mudança Média \\
Jordan (2003) & Mais de 25\%, menos de 35\% & Mudança Média \\
Kim (2005) & Mais de 25\%, menos de 35\% & Ano anterior \\
Yoo (2007) & Mais de 7,6\%, sob 22,4\% & Mudança Média \\
Danziger (1976) & Mais de 5\%, menos de 15\% & Mudança Média
\end{tabular}

Fonte: Adaptada de "Applying the incrementalism model to the free school meals policy in Korea," de Y. O. Chon e K. C. Ahn, 2015, International Review of Public Administration, 20, p. 196.

Para Chon e Ahn (2015), o Incrementalismo se baseia nos limites da capacidade cognitiva humana e na estabilidade do sistema de tomada de decisões. Com o incrementalismo, uma 
decisão é feita por intermédio de um processo pluralista de interação e adaptação mútua entre uma multiplicidade de atores fazendo pequenas mudanças (Chon e Ahn, 2015). Tanto é que um processo orçamentário caracterizado pelo incrementalismo pode ser associado ao consenso, enquanto grandes mudanças são associadas ao dissenso (Wildavsky, 1992).

Nesse sentido, percebe-se que o incrementalismo no orçamento se caracteriza como um processo mais constante, dado que as mudanças realizadas tendem a ser menores. Com pequenas variações incrementais, considera-se o orçamento do ano anterior como base, que é então gradualmente atualizado.

\subsection{PROCESSO ORÇAMENTÁRIO}

O orçamento público é o instrumento utilizado para a ação planejada do Estado, seja para manutenção de suas atividades, seja para execução de projetos, além de ser o instrumento do Poder Público capaz de expressar, para determinados períodos ou programas, a origem e quantia de recursos a serem obtidos, bem como informações sobre a destinação (Piscitelli \& Timbó, 2019). A publicação desse instrumento deve ser periódica e abarcar elementos acerca dos recebimentos e gastos realizados pelo gestor público. Isso possibilita aos cidadãos terem acesso a informações sobre como os recursos estão sendo utilizados e, com isso, exercerem seus direitos de fiscalização sobre os atos emanados do Poder Público.

Na elaboração, negociação e implementação do orçamento público, a ponderação de interesses, a barganha sobre possibilidades e o balizamento de representações sociais e políticas são fundamentais. Nas últimas décadas, tem havido enorme busca pela racionalização desse processo e do enquadramento do orçamento apenas como instrumento de gestão, que nunca deixará de ser também um instrumento político de ajustes entre fundos tributários e oferta de serviços e produtos para distintos setores da sociedade (Peres \& Mattos, 2017).

Em termos gerais, o processo orçamentário consiste em uma programação ou planejamento de despesas futuras, resultado de escolhas sociais feitas no presente, com base em expectativas quanto à evolução da receita de tributos e ao comportamento da taxa de inflação no período da realização do gasto governamental orçado. Expectativas divergentes quanto ao volume de tributos arrecadados e ao comportamento geral dos preços, bem como preferências distintas acerca dos objetivos dos gastos e tipos de despesas produzem tensões entre grupos de interesses concernentes à formulação e execução orçamentária, transformando o arcabouço legal do orçamento no resultado tangível das negociações entre grupos (Fittipaldi et al., 2017). 


\subsection{ESTUDOS ANTERIORES}

\section{Partindo do contexto do incrementalismo e equilíbrio pontuado, a Tabela 2 apresenta} alguns estudos correlatos à temática pesquisada:

Tabela 2

Estudos anteriores usando o incrementalismo ou o equilibrio pontuado

\begin{tabular}{|c|c|c|}
\hline Autores & Objetivo & Resultados \\
\hline True (2000) & $\begin{array}{l}\text { Buscou demonstrar que o incrementa- } \\
\text { lismo não explica de forma abrangen- } \\
\text { te a tomada de decisões do governo e } \\
\text { defende a incorporação do incremen- } \\
\text { talismo em uma teoria mais ampla da } \\
\text { formulação de políticas governamen- } \\
\text { tais. }\end{array}$ & $\begin{array}{l}\text { Afirmaram que o incrementalismo fracassa na expli- } \\
\text { cação abrangente das mudanças nas políticas e no } \\
\text { orçamento, mas é útil quando incorporado à teoria } \\
\text { do equilíbrio pontuado e ao modelo de orçamento } \\
\text { de avalanches. Mudanças em larga escala são uma } \\
\text { parte regular e significativa da tomada de decisões } \\
\text { orçamentárias em nível nacional. }\end{array}$ \\
\hline $\begin{array}{l}\text { John e Mar- } \\
\text { getts (2003) }\end{array}$ & $\begin{array}{l}\text { Realizaram a análise de alterações } \\
\text { percentuais do orçamento nos orça- } \\
\text { mentos do governo central para verifi- } \\
\text { car se a distribuição é incrementalista } \\
\text { ou de equilíbrio pontuado. }\end{array}$ & $\begin{array}{l}\text { Verificaram que as mudanças no orçamento formam } \\
\text { uma distribuição leptocúrtica, ou seja, em acordo } \\
\text { com a hipótese do modelo de equilíbrio pontuado. } \\
\text { Esse padrão aparece na maioria dos setores de polí- } \\
\text { ticas analisados. }\end{array}$ \\
\hline
\end{tabular}

Analisaram o orçamento francês para

Baumgartner, testar atrito, incrementalismo e equi-

Foucault. líbrio pontuado, com dados incluindo

e François o orçamento geral do estado desde

(2006) 1820; orçamentos ministeriais para sete ministérios desde 1868; e uma série com dez ministérios desde 1947.

Os resultados são de que o orçamento francês é extremamente pontuado. Os dados sugerem que a mais importante força motriz do equilíbrio pontuado é a arquitetura da cognição humana, e não qualquer característica institucional específica dos governos.

(a)

Examinaram se o Orçamento Federal dos Estados Unidos da América

Anderson e Harbridge (2010) (EUA) é incremental. Tal análise foi realizada pelo pressuponto de que o orçamento daquele País é estável e que um orçamento incremental é caracterizado como estável.

Estudou a evolução dos principais tipos de gastos da União Europeia (1984-2011), avaliando o padrão de estabilidade política e mudança pela luz do incrementalismo e equilíbrio pontuado.
Os resultados indicam que a baixa proporção das mudanças é realmente pequena. Na maior parte dos anos, mais de $60 \%$ das alterações orçamentais são maiores do que $5 \%$, e cerca da metade é maior do que $10 \%$, caracterizando o orçamento como incremental. $O$ incrementalismo tem aumentado ao longo do tempo, mas ainda mais de $20 \%$ das mudanças são caracterizadas por mudanças maiores que $50 \%$.

A análise mostra que as políticas da UE não evoluem seguindo um padrão incrementalista, mas por uma dinâmica de equilíbrio pontuado. As políticas da UE tendem a seguir uma dinâmica geral de estabilidade e ajustes incrementais, caracterizada por mudanças nas políticas que permanecem dentro de uma "faixa de flutuação" de mais ou menos 10\%. Porém, em algumas ocasiões, esse padrão de estabilidade é interrompido, levando a "pontuações" ou alterações políticas não incrementais.

Indicou um padrão de distribuição das variações com períodos de estabilidade e pequenas interrupções. As maiores amplitudes de variações ocorreram nos anos 1986, 1989 e 1990, coincidindo com períodos de significativas mudanças institucionais. Da mesma forma, a redução na amplitude das variações observadas a partir de 1996 pode indicar alinhamento com a estabilização monetária como fator institucional que contribui para o equilíbrio da execução orçamentária.
Investigou o comportamento de estabilidade e mudança na execução orçamentária da União, entre 1980 e 2014, buscando verificar a aderência da Teoria do Equilíbrio Pontuado na execução das políticas públicas por meio do orçamento federal.

(2018)




\begin{tabular}{lll}
\hline \multicolumn{1}{c}{ Autores } & \multicolumn{1}{c}{ Objetivo } & \multicolumn{1}{c}{ Resultados } \\
\hline & $\begin{array}{l}\text { Pesquisaram o gasto público por } \\
\text { função no Brasil. Utilizaram-se de } \\
\text { medidas de desigualdade para a } \\
\text { alttipaldi et }\end{array}$ & $\begin{array}{l}\text { Observaram no estudo que o gasto social apresentou } \\
\text { um padrão incremental, no período de 1995 a 2014, } \\
\text { e que essa característica se reproduzirá no médio } \\
\text { prazo. }\end{array}$ \\
& da variável de interesse. &
\end{tabular}

\begin{tabular}{|c|c|c|}
\hline $\begin{array}{l}\text { Guragain e } \\
\text { Lim (2018) }\end{array}$ & $\begin{array}{l}\text { Analisaram empiricamente os dados } \\
\text { das séries temporais orçamentárias do } \\
\text { Nepal de } 1990 \text { a } 2017 \text {. }\end{array}$ & $\begin{array}{l}\text { Concluíram que o padrão de apropriação do } \\
\text { orçamento é caracterizado por várias flutuações e } \\
\text { mudanças de políticas que não se limitam a mudan- } \\
\text { ças incrementais. Os autores consideram as hipóteses } \\
\text { sobre mudança incremental e equilíbrio pontuado, } \\
\text { em que três hipóteses adicionais, baseadas em } \\
\text { suposições de ajuste orçamentário, são derivadas e } \\
\text { avaliadas: pontuação paralela, sequencial e comple- } \\
\text { mentar. }\end{array}$ \\
\hline $\begin{array}{l}\text { Silvestre e } \\
\text { Araújo (2015) }\end{array}$ & $\begin{array}{l}\text { Procuraram perceber em que medida } \\
\text { a distribuição dos orçamentos aos go- } \\
\text { vernos locais do Ceará sofre (ou não) } \\
\text { variações. Em um segundo momento, } \\
\text { tentaram depreender quais as recei- } \\
\text { tas de cada um dos municípios que } \\
\text { melhor descrevem e auxiliam a opção } \\
\text { política de investimento local. }\end{array}$ & $\begin{array}{l}\text { Considerando o período de } 2006 \text { a } 2010 \text {, concluíram } \\
\text { que nos governos locais do Ceará o investimento } \\
\text { público apresenta períodos de estabilidade seguido por } \\
\text { períodos de grande alteração dos orçamentos. Essas } \\
\text { conclusões confirmam os pressupostos da teoria do } \\
\text { equilíbrio pontuado. Conclui-se igualmente que o inves- } \\
\text { timento público que vem sendo realizado é dependente } \\
\text { das transferências que a União faz para os governos } \\
\text { locais do Estado do Ceará e das receitas de capital. }\end{array}$ \\
\hline $\begin{array}{l}\text { Zapelini, } \\
\text { Lima, e Gue- } \\
\text { des (2017) }\end{array}$ & $\begin{array}{l}\text { Averiguaram a evolução histórica da } \\
\text { política habitacional brasileira entre } \\
1967 \text { e } 2014 \text { por meio da teoria do } \\
\text { equilíbrio pontuado. Para tanto, foram } \\
\text { estudadas as variáveis: orçamento, } \\
\text { produção de leis pelo Poder Legislati- } \\
\text { vo e atenção dedicada ao assunto pe- } \\
\text { los meios de comunicação de massa. }\end{array}$ & $\begin{array}{l}\text { Constataram que existem períodos em que o equi- } \\
\text { líbrio da política é interrompido por pontuações } \\
\text { relevantes. Verificaram que o orçamento federal para } \\
\text { a área, durante o período estudado, não possui uma } \\
\text { trajetória incremental, percebendo-se significativo de- } \\
\text { clínio no final da década de } 1980 \text { em diante, mesmo } \\
\text { com a instituição do Programa Minha Casa Minha } \\
\text { Vida. }\end{array}$ \\
\hline
\end{tabular}

Pode-se dizer, a partir das análises dos estudos da Tabela 2, que as mudanças observadas não se limitam a incrementais ou de equilíbrio pontuado, mas de alternância desses padrões. Considerando o cenário nacional, de forma geral, percebe-se que o orçamento tem mudança incremental com indicativo de permanência desse tipo de mudança no curto prazo, mas também se nota que períodos de mudanças institucionais se caracterizaram como fatores determinantes mais relevantes do que ciclos políticos para que tais mudanças sejam associadas ao equilíbrio pontuado (Carvalho, 2018; Fittipaldi et al., 2017). Por outro lado, a análise dos estudos internacionais permite afirmar que a dinâmica do orçamento observada em locais como Estados Unidos e União Europeia segue a teoria do equilíbrio pontuado.

\section{METODOLOGIA}

Em termos de enquadramento metodológico, este estudo se caracteriza como descritivo no que se refere aos objetivos, pois visa analisar se o processo orçamentário dos governos locais paranaenses segue os padrões de mudança incremental nas dotações orçamentárias 
nacionais, ou a ocorrência de pontuações orçamentárias, caracteriza-se como quantitativa, em razão do fato de que os montantes das variações orçamentárias é que determinam se as mudanças são incrementais ou de pontuação. (Richardson \& Peres, 1999).

Este estudo foi realizado no contexto dos municípios paranaenses. A população corresponde aos governos locais dos 399 municípios, dos quais foram coletados dados dos 55 maiores municípios paranaense, classificados conforme quantidades de habitantes informados pelo Instituto Brasileiro de Geografia e Estatística (IBGE, 2016). Na sequência, foram analisados e excluídos os municípios que não tinham todas as variáveis em comum, o que resultou em 39 municípios correspondentes aos governos locais, compondo a amostra do estudo (Tabela 3).

Destaca-se que os dados dos municípios foram levantados segundo o eixo temporal 2014 a 2017, e que a coleta destes ocorreu por meio do Siconfi, no mês de outubro de 2018. O recorte foi definido em razão de a disponibilização das informações dos municípios ter ocorrido em 2013 e pelo fato de que nem todos apresentavam os dados das despesas do ano de 2013. Além disso, o recorte possibilita a análise do orçamento aprovado pelos eleitos em 2012 , bem como dos anos subsequentes.

Tabela 3

Amostra da pesquisa

\begin{tabular}{ccclcl}
\hline Ordem & Governo local & Ordem & Governo local & Ordem & Governo local \\
\hline 1 & Almirante Tamandaré & 14 & lbiporã & 27 & Pinhais \\
2 & Apucarana & 15 & lrati & 28 & Ponta Grossa \\
3 & Arapongas & 16 & Lapa & 29 & Prudentópolis \\
4 & Assis Chateaubriand & 17 & Londrina & 30 & Rio Negro \\
5 & Campina Grande do Sul & 18 & Mandaguari & 31 & Rolândia \\
6 & Campo Largo & 19 & Marechal Cândido Rondon & 32 & Santo Antônio da Platina \\
7 & Cascavel & 20 & Maringá & 33 & São José dos Pinhais \\
8 & Castro & 21 & Matinhos & 34 & São Mateus do Sul \\
9 & Colombo & 22 & Palmas & 35 & Sarandi \\
10 & Cornélio Procópio & 23 & Palmeira & 36 & Telêmaco Borba \\
11 & Curitiba & 24 & Paranaguá & 37 & Toledo \\
12 & Foz do lguaçu & 25 & Paranavaí & 38 & Umuarama \\
13 & Guarapuava & 26 & Pato Branco & 39 & União da Vitória \\
\hline
\end{tabular}

Com o propósito de analisar se o processo orçamentário dos governos locais paranaenses segue os padrões de mudança incremental nas dotações orçamentárias ou a ocorrência de pontuações orçamentárias, foram estabelecidas quatro variáveis de análise: educação, saúde, segurança pública e urbanismo, conforme descrito na Tabela 4 : 
Tabela 4

Variáveis da pesquisa

\begin{tabular}{|c|c|c|}
\hline Variáveis & Conceito operacional & Referências \\
\hline Educação & $\begin{array}{l}\text { Valores de receitas alocadas a despesas com } \\
\text { educação. }\end{array}$ & $\begin{array}{l}\text { Carvalho (2018), Fittipaldi et al. } \\
\text { (2017) e Guragain e Lim (2018). }\end{array}$ \\
\hline Saúde & $\begin{array}{l}\text { Valores de receitas alocadas a despesas com } \\
\text { saúde. }\end{array}$ & $\begin{array}{l}\text { Carvalho (2018), Fittipaldi et al. } \\
\text { (2017) e Guragain e Lim (2018). }\end{array}$ \\
\hline Segurança pública & $\begin{array}{l}\text { Valores de receitas alocadas a despesas com } \\
\text { segurança pública. }\end{array}$ & Guragain e Lim (2018). \\
\hline Urbanismo & $\begin{array}{l}\text { Valores de receitas alocadas a despesas com } \\
\text { urbanismo. }\end{array}$ & Carvalho (2018). \\
\hline
\end{tabular}

A variável saúde são alocações orçamentárias para essa despesa específica. Nos modelos de crescimento endógeno seria ponderada como saúde preventiva, a qual influenciaria o crescimento econômico por garantir a permanência do cidadão no mercado de trabalho (Ashwani \& Sheera, 2017).

A variável educação são alocações orçamentárias referentes às despesas com educação, correspondente à responsabilidade do governo local para essa área específica nos municípios. Zanmaria e Castilho (2006) consideram que uma boa qualidade educacional minimizaria as disparidades que alteram o bem-estar social, fornecendo mecanismo para a capacitação dos cidadãos e sua inserção no mercado, também impactando o crescimento econômico.

A variável de urbanismo justifica-se pelas alocações orçamentárias a essa área, representando externalidades positivas que podem ser absorvidas por uma parte significativa da economia local, impulsionando o bem-estar econômico da sociedade (Barro, 1990).

Por fim, a variável segurança pública representa alocações orçamentárias relevantes para o governo local com reflexos na sociedade como um todo, proporcionando a paz e a tranquilidade para os residentes dos municípios. Com a finalidade de atender ao objetivo desta pesquisa, na análise contou-se com o auxílio do programa Excel para a elaboração de estatísticas descritivas e gráficos.

\section{RESULTADOS E DISCUSSÃO}

$\mathrm{Na}$ Tabela 5 evidenciam-se as quantidades de variações da alocação orçamentária por variáveis analisadas, bem como foram observados oito tamanhos de variações, as quais também foram relacionadas a variações negativas ou redutoras e positivas ou aumentativas. 
Tabela 5

Quantidade de variações da execução orçamentária por variáveis entre 2014 e 2017, por tamanho da variação

\begin{tabular}{|c|c|c|c|c|c|c|c|c|}
\hline Variáveis & $\begin{array}{l}\text { Redução } \\
\text { maior que } \\
50 \%\end{array}$ & $\begin{array}{l}\text { Redução } \\
\text { maior } \\
\text { que } 30 \% \\
\text { e menor } \\
\text { ou igual } \\
\text { a } 50 \%\end{array}$ & $\begin{array}{l}\text { Redução } \\
\text { maior } \\
\text { que } 10 \% \\
\text { e menor } \\
\text { ou igual } \\
\text { a } 30 \%\end{array}$ & $\begin{array}{l}\text { Redução } \\
\text { menor ou } \\
\text { igual a } 10 \% \\
\text { ou aumento } \\
\text { menor ou } \\
\text { igual a } 10 \%\end{array}$ & $\begin{array}{l}\text { Aumento } \\
\text { maior } \\
\text { que } 10 \% \\
\text { e menor } \\
\text { ou igual } \\
\text { a } 30 \%\end{array}$ & $\begin{array}{l}\text { Aumento } \\
\text { maior } \\
\text { que } 30 \% \\
\text { e menor } \\
\text { ou igual a } \\
50 \%\end{array}$ & $\begin{array}{l}\text { Aumento } \\
\text { maior } \\
\text { que } 50 \% \\
\text { e menor } \\
\text { ou igual } \\
\text { a } 200 \%\end{array}$ & $\begin{array}{c}\text { Aumento } \\
\text { maior } \\
\text { que } \\
200 \%\end{array}$ \\
\hline Educação & 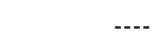 & $\cdots$ & 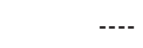 & 61 & 55 & 1 & $-\cdots$ & $\cdots$ \\
\hline Saúde &.-- & 1 & 1 & 56 & 57 & 2 & $\cdots$ & --- \\
\hline $\begin{array}{l}\text { Segurança } \\
\text { pública }\end{array}$ & 12 & 7 & 14 & 35 & 19 & 8 & 15 & 7 \\
\hline Urbanismo & 3 & 13 & 24 & 34 & 19 & 9 & 14 & 1 \\
\hline
\end{tabular}

Os resultados demonstrados na Tabela 5 indicam mudanças, de um ano para o outro, relacionadas à execução de despesas. As funções segurança pública e urbanismo, em uma quantidade de 8 e 9 governos locais, respectivamente, apresentaram uma concentração na variação da alocação orçamentária que se enquadra na categoria "maior que $30 \%$ e menor ou igual a $50 \%$ " de aumento. Ainda nessas duas funções, respectivamente, houve uma quantidade de 15 e 14 dos governos locais com uma variação na categoria "maior que 50\% e menor ou igual a $200 \%$ " de aumento na alocação do orçamento nesses mesmos setores.

Nessas observações, as mudanças significativas no orçamento do governo local seguem a ocorrência simultânea de pontuações nas alocações orçamentárias em muitos setores. Tais observações também presumem que a tendência de mudanças incrementais na apropriação do orçamento será interrompida por pontuações em determinados momentos.

$\mathrm{Na}$ Tabela 6 apresentam-se dados relativos às estatísticas descritivas aplicadas em cada variável contida no construto da pesquisa. Foram determinados: desvio padrão, máximo, média, mediana e mínimo das variáveis investigadas.

Tabela 6

Estatística descritiva

\begin{tabular}{llcrr}
\hline Estatística descritiva & & $\mathbf{2 0 1 5}$ & $\mathbf{2 0 1 6}$ & $\mathbf{2 0 1 7}$ \\
\hline & Desvio padrão & $6 \%$ & $6 \%$ & $5 \%$ \\
Educação & Máximo & $34 \%$ & $25 \%$ & $28 \%$ \\
& Média & $13 \%$ & $10 \%$ & $7 \%$ \\
& Mediana & $14 \%$ & $11 \%$ & $5 \%$ \\
& Mínimo & $-5 \%$ & $-10 \%$ & $-5 \%$ \\
\hline
\end{tabular}




\begin{tabular}{|c|c|c|c|c|}
\hline & & 2015 & 2016 & 2017 \\
\hline & Desvio padrão & $7 \%$ & $9 \%$ & $8 \%$ \\
\hline & Máximo & $27 \%$ & $36 \%$ & $31 \%$ \\
\hline \multirow[t]{5}{*}{ Saúde } & Média & $9 \%$ & $12 \%$ & $10 \%$ \\
\hline & Mediana & $8 \%$ & $12 \%$ & $8 \%$ \\
\hline & Mínimo & $-16 \%$ & $-7 \%$ & $-34 \%$ \\
\hline & Desvio padrão & $85 \%$ & $51 \%$ & $63 \%$ \\
\hline & Máximo & $903 \%$ & $291 \%$ & $612 \%$ \\
\hline \multirow[t]{5}{*}{ Segurança } & Média & $46 \%$ & $35 \%$ & $21 \%$ \\
\hline & Mediana & $8 \%$ & $12 \%$ & $-1 \%$ \\
\hline & Mínimo & $-96 \%$ & $-78 \%$ & $-91 \%$ \\
\hline & Desvio padrão & $38 \%$ & $31 \%$ & $17 \%$ \\
\hline & Máximo & $171 \%$ & $229 \%$ & $53 \%$ \\
\hline \multirow[t]{3}{*}{ Urbanismo } & Média & $23 \%$ & $15 \%$ & $-14 \%$ \\
\hline & Mediana & $21 \%$ & $7 \%$ & $-16 \%$ \\
\hline & Mínimo & $-82 \%$ & $-62 \%$ & $-54 \%$ \\
\hline
\end{tabular}

Confirmando os resultados da Tabela 5, as variáveis segurança pública e urbanismo apresentaram estatísticas descritivas elevadas conforme se expôs na Tabela 6. A alta variabilidade dessas medidas, dada a observação do desvio padrão, indica que há diferenças significativas para as alocações orçamentárias referentes aos anos anteriores dos respectivos governos locais analisados.

Na sequência (Figuras 1 a 4 ) apresentam-se os resultados da variação percentual na apropriação do orçamento de cada variável, ilustrados pelas apresentações gráficas.

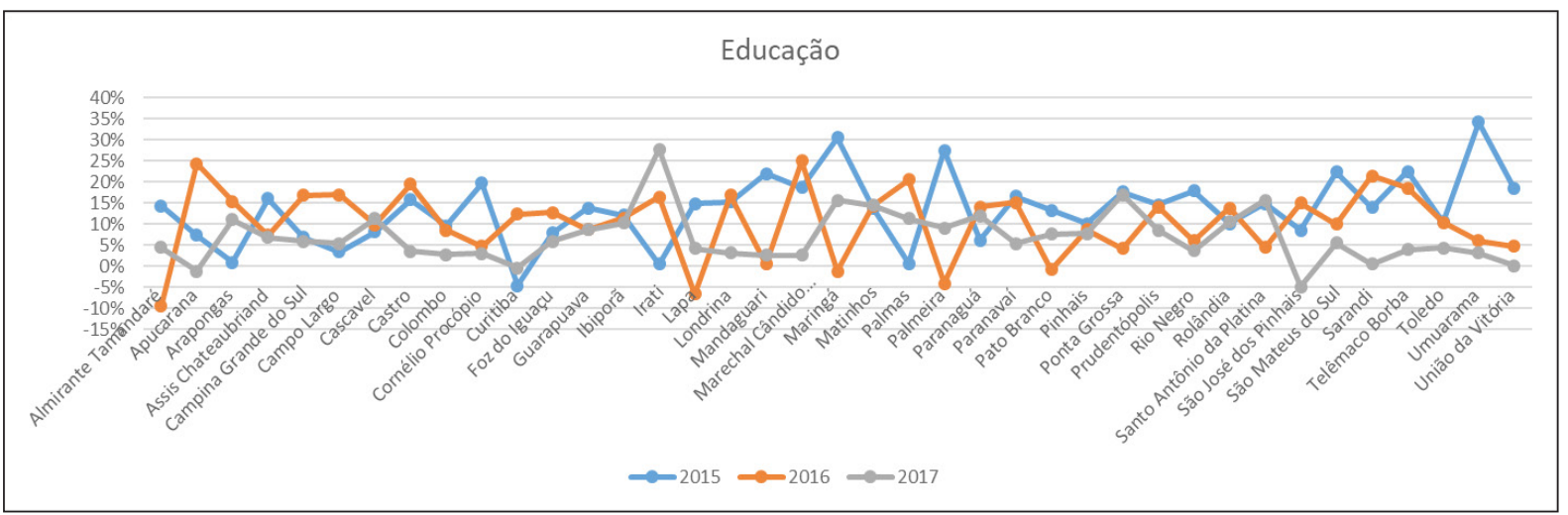

Figura 1. Variação percentual na apropriação do orçamento da educação.

Observando a Figura l, os anos 2015 e 2017 mostram os resultantes da variável educação, os quais são caracterizados por flutuações. Assim, argumenta-se que o orçamento anual destinado a esse setor seguiu tendências incrementais, sendo interrompido por 
pontuações em determinados momentos e também com mudanças significativas na variação orçamentária dos governos locais, induzindo a ocorrência de pontuações simultâneas nas alocações orçamentárias na função (variável) analisadas.

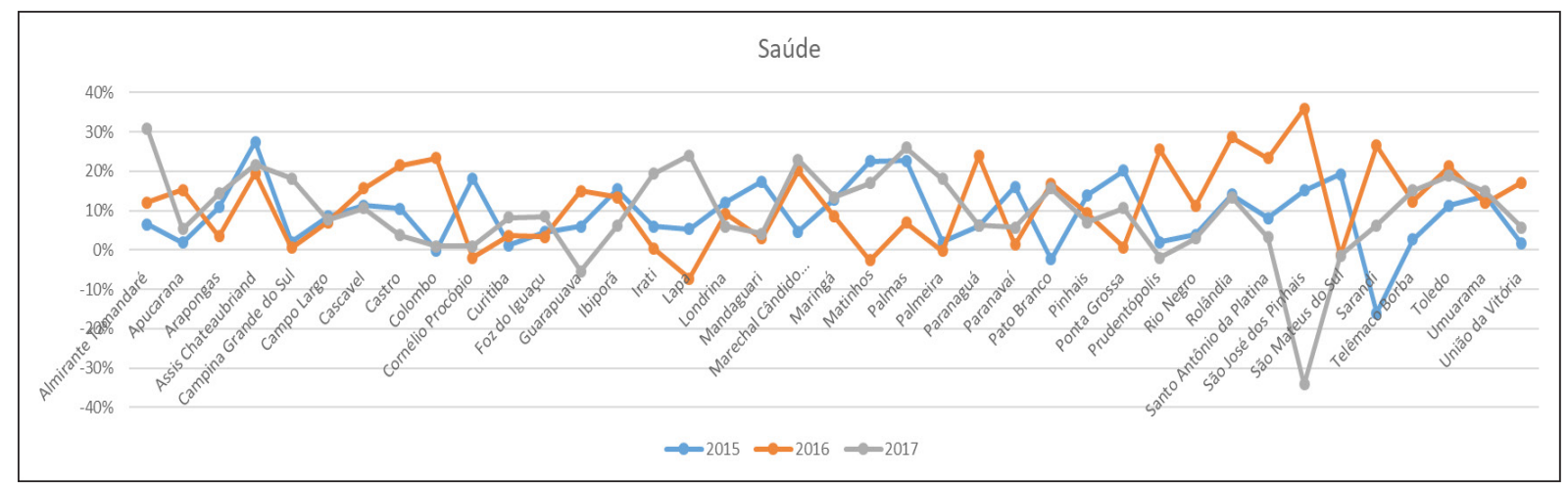

Figura 2. Variação percentual na apropriação do orçamento da saúde.

Na Figura 2 destaca-se que em todos os anos os resultantes da variável saúde não foram constantes.

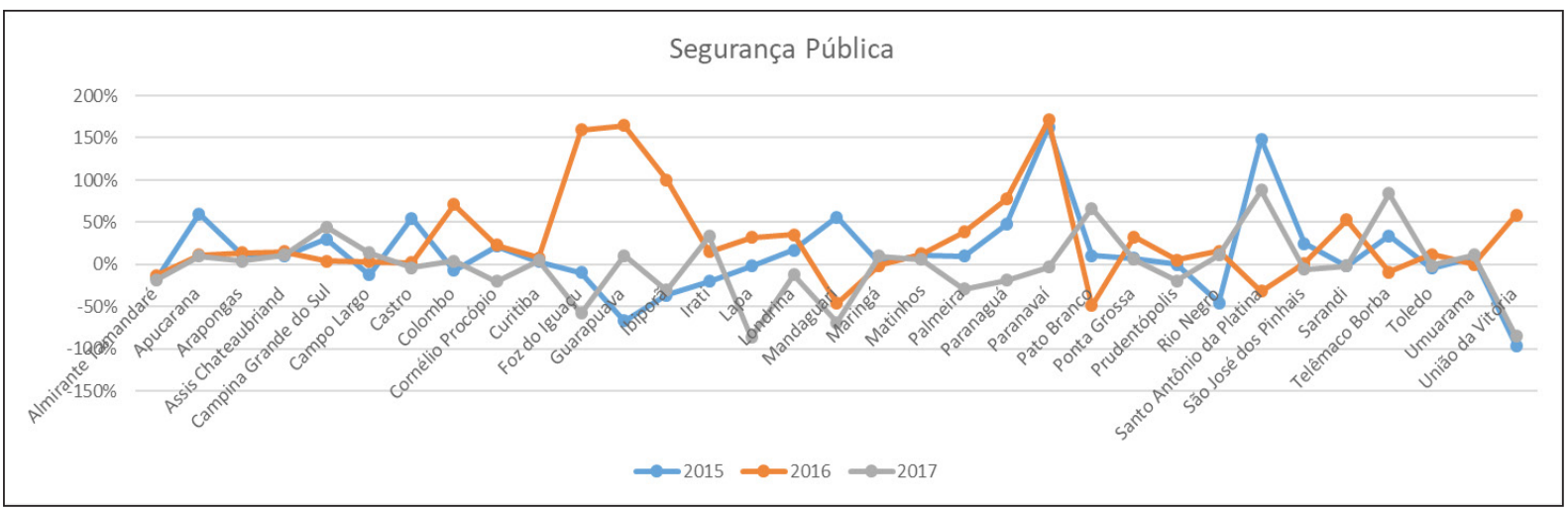

Figura 3. Variação percentual na apropriação do orçamento da segurança pública.

Da interpretação da Figura 3 é possível dizer que em todos os anos os resultados da variável segurança pública não foram lineares, mudanças incrementais na apropriação do orçamento foram interrompidas por pontuações em determinados momentos, ocorrendo também mudanças significativas na variação orçamentária do governo local, induzindo a ocorrência de pontuações simultâneas nas alocações orçamentárias nas funções orçamentárias analisadas.

Por fim, a Figura 4 refere-se à variação percentual na apropriação do orçamento para a função urbanismo: 


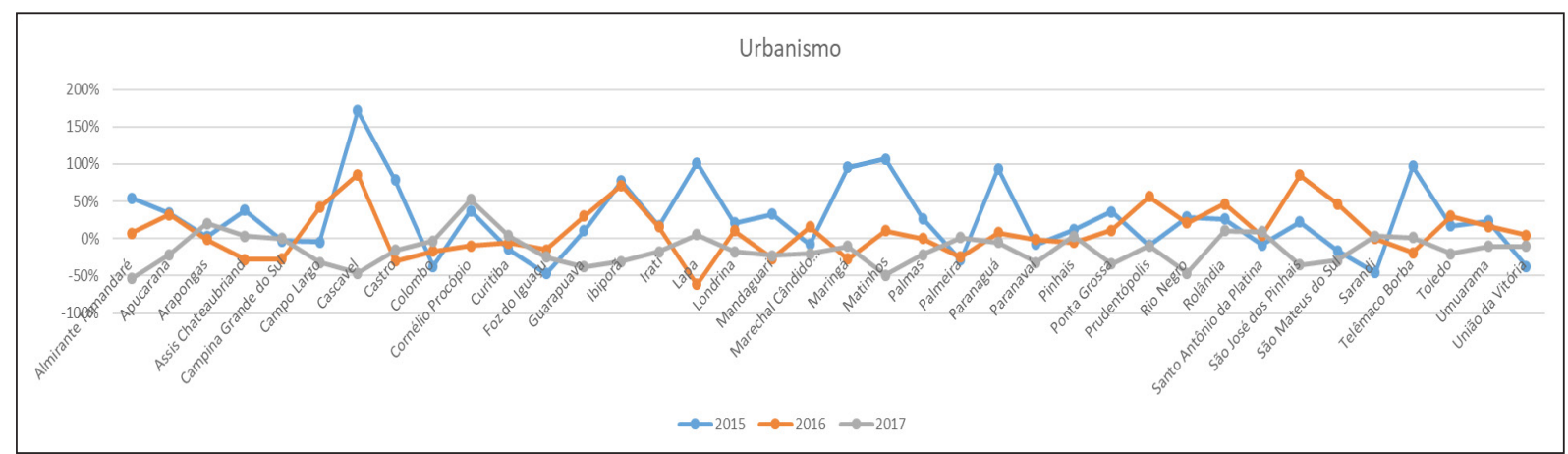

Figura 4. Variação percentual na apropriação do orçamento do urbanismo.

Da análise da Figura 4 é possível destacar que, como na Figura 3, em todos os anos as flutuações resultantes da variável urbanismo não foram uniformes. Dessa forma, argumenta-se que a tendência de mudanças incrementais na apropriação do orçamento no período analisado foi interrompida por pontuações em determinados momentos, e que houve mudanças significativas na variação orçamentária do governo local, percebendo-se a ocorrência de pontuações simultâneas nas alocações orçamentárias entre os setores das políticas públicas concernentes às funções orçamentárias pesquisadas.

De modo geral, as variações percentuais nas alocações de despesa nos anos de 2014, 2015, 2016 e 2017 foram pontuadas positiva e negativamente, indo ao encontro de Carvalho (2018) e de Guragain e Lim (2018). Por meio dos resultados deste estudo se percebe um padrão de equilíbrio com interrupções, corroborando a hipótese central de que a Teoria do Equilíbrio Pontuado é válida para analisar o comportamento das variações orçamentárias, em especial no caso do presente estudo, com os governos locais paranaenses.

Com base nos elementos analisados, os resultados também convergem com os achados de Zapelini et al. (2017), que realizaram estudos acerca da evolução histórica da política habitacional brasileira entre 1967 e 2014 com a lente da Teoria do Equilíbrio Pontuado. Contudo, ressalta-se que os resultados são divergentes dos achados de Anderson e Harbridge (2010) e Fittipaldi et al. (2017). Anderson e Harbridge examinaram o orçamento dos Estados Unidos e indicaram pequenas mudanças orçamentais analisadas por qualquer lógica padrão, enquanto Fittipaldi et al. observaram que o gasto social apresentou um padrão incremental, no período de 1995 a 2014 no Brasil, característica esta que se reproduziria no médio prazo.

\section{CONSIDERAÇÕES FINAIS}

Este estudo ofereceu uma sinopse das apropriações orçamentárias nos governos locais paranaenses, bem como examinou a aplicabilidade da teoria do equilíbrio pontuado em economias politicamente instáveis e em desenvolvimento. 
Embasando o estudo pela variação de Baumgartner e Jones (1993), em que os autores determinam $\pm 25 \%$ em relação ao orçamento do ano anterior para explicar a pontuação, os aspectos da variação percentual nos dados de apropriação do orçamento são distribuídos de forma não normal, conforme ilustrado pelas apresentações gráficas e estatísticas descritivas, o que sugere a ocorrência de um padrão de equilíbrio pontuado.

Face aos argumentos apresentados, a análise dos dados justifica esse padrão de apropriações orçamentárias nos governos locais paranaenses, e os resultados são caracterizados por flutuações, o que indica que as mudanças na política não se limitam a mudanças incrementais.

Apoderando-se dos aspectos observados, conclui-se que, de modo geral, o processo orçamentário dos governos locais paranaenses segue com ocorrência de pontuações, que pressupõem que a tendência de mudanças incrementais na apropriação do orçamento será interrompida por pontuações em determinados momentos e também haverá a ocorrência de pontuações simultâneas nas alocações orçamentárias entre os setores das políticas públicas.

As investigações desta pesquisa contribuem para diversas áreas, como Políticas Públicas, Administração Pública, Gestão Pública, Contabilidade Pública e Contabilidade Gerencial. Assim, a análise das dinâmicas orçamentárias dos governos locais paranaenses estende a literatura acerca da temática, corroborando Carvalho (2018), o qual acredita que o desenvolvimento dessa nova agenda de pesquisa representa um avanço nos estudos de políticas públicas e orçamento no Brasil, uma vez que grande parte dos estudos orçamentários não utiliza modelos teóricos para examinar e compreender o comportamento das decisões no processo orçamentário.

Este estudo apresenta limitações em razão das decisões dos pesquisadores a respeito do delineamento metodológico, entretanto, essas limitações possibilitam a oportunidade de pesquisas futuras. Uma delas seria a amostra, por exemplo, de ampliar os governos locais paranaenses analisados; outra seria analisar mais variáveis, como habitação, saneamento básico, turismo, etc.; além disso, sugere-se a utilização de um recorte longitudinal maior e também a aplicação de modelos com testes estatísticos.

\section{REFERÊNCIAS}

Alves, M. A. (2016). O modelo incremental como teoria para o processo orçamentário. Revista Brasileira de Planejamento e Orçamento, 6(2), 124-137.

Anderson, S., \& Harbridge, L. (2010). Incrementalism in appropriations: Small aggregation, big changes. Public Administration Review, 70(3), 464-474. 
Ashwani, B., \& Sheera, V. P. (2017). Public spending and economic growth for Indian states. International Journal of Public Sector Performance Management, 3(3), 250-265.

Bailey, J. J., \& O'connor, R. J. (1975). Operationalizing incrementalism: Measuring the muddles. Public Administration Review, 60-66.

Barro, R. J. (1990, October). Government spending in a simple model of endogenous growth. Journal of Political Economy, 98, 103-125.

Baumgartner, F. R., \& Jones, B. D. (1993). Agendas and instability in American politics. Chicago, IL: University of Chicago Press.

Baumgartner, F. R., \& Jones, B. D. (2010). Agendas and instability in American politics. Chicago, IL: University of Chicago Press.

Baumgartner, F. R., Foucault, M., \& François, A. (2006). Punctuated equilibrium in French budgeting processes. Journal of European Public Policy, 13(7), 1086-1103.

Carvalho, D. I. (2018). Teoria do Equilíbrio Pontuado: Uma Análise da Execução Orçamentária no Brasil no Período de 1980-2014. Revista do Serviço Público, 69(1), 85-110.

Chon, Y. O, \& Ahn, K. C. (2015). Applying the incrementalism model to the free school meals policy in Korea. International Review of Public Administration, 20(2), 194-207.

Citi, M. (2013). EU budgetary dynamics: incremental or punctuated equilibrium? Journal of European Public Policy, 20(8), 1157-1173.

Danziger, J. N. (1976). Assessing incrementalism in British municipal budgeting. British Journal of Political Science, 6(3), 335-350.

Fittipaldi, I., Costa, S. F., \& Araújo, C. M. C. de. (2017). O gasto público federal brasileiro: Um perfil incrementalista? Revista do Serviço Público, 68(3), 611-630.

Givel, M. (2010). The evolution of the theoretical foundations of punctuated equilibrium theory in public policy. Review of Policy Research, 27(2), 187-198.

Guragain, H. P., \& Lim, S. (2018). Nepalese Budgetary Dynamics: Following Incrementalism or Punctuated Equilibrium? Public Organization Review, 1-26.

Instituto Brasileiro de Geografia e Estatística. (2016). População. Recuperado de https:// www.ibge.gov.br/ 
John, P., \& Margetts, H. (2003). Policy punctuations in the UK: Fluctuations and equilibria in central government expenditure since 1951. Public Administration, 81(3), 411-432.

Jones, B. D., \& Baumgartner, F. R. (2012). From there to here: Punctuated equilibrium to the general punctuation thesis to a theory of government information processing. Policy Studies Journal, 40(1), 1-20.

Jones, B. D., True, J., \& Baumgartner, F. (2006). Punctuated Equilibrium Theory. Retrieved from https://paulcairney.files.wordpress.com/2017/07/true-baum-and-jones-2007-in-sabatier.pdf

Jordan, M. M. (2003). Punctuations and agendas: A new look at local government budgetexpenditures. Journal of Policy Analysis and Management, 22(3), 345-360.

Kim, C.-H. (2005). A study on patterns determinants of the change of government expenditure: Focused on the change of functional expenditure of Korean central government. KPAR, 39(3), 115-136.

Kuhlmann, J., \& Van Der Heijden, J. (2018). What Is Known about Punctuated Equilibrium Theory? And What Does That Tell Us about the Construction, Validation, and Replication of Knowledge in the Policy Sciences? Review of Policy Research, 35(2), 326-347.

Ollaik, L. G., \& Medeiros, J. J. (2011). Instrumentos governamentais: Reflexões para uma agenda de pesquisas sobre implementação de políticas públicas no Brasil. Revista de Administração Pública, 45(6), 1943-1967.

Peres, U. D., \& Mattos, B. B. (2017). A Participação Social e o Conflito Distributivo na Planificação e Orçamentação Públicas: O Caso do Município de São Paulo. Cadernos Gestão Pública e Cidadania, 22(73), 456-477.

Piscitelli, R. B., \& Timbó, M. Z. (2019). Contabilidade pública: Uma abordagem da administração financeira pública. $14^{\mathrm{a}}$ ed.). São Paulo: Atlas.

Richardson, R. J., \& Peres, J. A. (1999). Pesquisa Social: Métodos e técnicas. São Paulo: Atlas.

Silvestre, H. C., \& Araújo, J. F. F. E. (2015). Teoria do Equilíbrio Pontuado nas Políticas Públicas Brasileiras: O Caso do Ceará. Revista de Administração Contemporânea, 19(6), 696 711.

Sistema de Informações Contábeis e Fiscais do Setor Público Brasileiro. (2018). Recuperado de https://siconfi.tesouro.gov.br/siconfi/pages/public/consulta_finbra/finbra_list.jsf 
True, J. L. (2000). Avalanches and incrementalism: Making policy and budgets in the United States. The American Review of Public Administration, 30(1), 3-18.

Wildavsky, A. B. (1964). Politics of the budgetary process. Boston: Little, Brown.

Wildavsky, A. B. (1992). The new politics of the budgetary process. 2nd ed.). New York: HarperCollins.

Yoo, K.-R. (2007). The time series analysis of disconnection policy about South Korea's central government budget. KPAR, 4l(2), 95-116.

Zanmaria, N. A., \& Castilho, M. L. (2006). Gastos públicos com educação e seus reflexos no crescimento econômico brasileiro. Revista Faz Ciência, 8(1), 123.

Zapelini, M. B., Lima, J. G., \& Guedes, M. C. (2017). Evolução da Política Habitacional no Brasil (1967-2014): Uma Análise de Equilíbrio Pontuado. Revista Interdisciplinar de Gestão Social, 6(3).

\section{Como citar este artigo:}

\section{ABNT}

SILVA, Cristiane Aparecida da et al. Dinâmicas orçamentárias dos governos locais paranaenses: seguindo o incrementalismo ou equilíbrio pontuado? RACE, Revista de Administração, Contabilidade e Economia, Joaçaba: Editora Unoesc, v. 19, n. 2, p. 299-316, maio/ago. 2020. Disponível em: http://editora.unoesc.edu.br/index.php/race. Acesso em: dia/mês/ano.

\section{APA}

Silva, C. A. da, Suave, R., Silva, F. A. da, \& Rosa, F. S. da. (2020). Dinâmicas orçamentárias dos governos locais paranaenses: Seguindo o incrementalismo ou equilíbrio pontuado? RACE, Revista de Administração, Contabilidade e Economia, 19(2), 299-316. Recuperado de $h+t p: / / e d i t o r a . u n o e s c . e d u . b r / i n d e x . p h p / r a c e$ 